\title{
NOTAS SOBRE NUMERALES Y EL PLURAL EN EL QUICHÉ DE SAJCABAJÅ
}

\section{Otto Schumann}

Centro de Estudios Mayas UNAM.

Este trabajo fue elaborado gracias a la ayuda de la Misión Francesa en Guatemala (Centre National de la Recherche Scientifique), y forma parte de una serie de trabajos que he de escribir, sobre el quiché de San Andrés Sajcabajá. El material para éstos fue recopilado durante mi estancia con la misión francesa en dicho lugar, en el mes de febrero de 1975 .

El quiché que se habla en Sajcabajá es un dialecto de dicha lengua, que presenta características propias, tanto de tipo fonológico, como léxico y sintáctico; los puntos que trato en este artículo son de interés por las razones siguientes: primero, que el sistema numeral del quiché casi siempre se presenta en su forma simplificada, dando sólo los cardinales sin presentar el sistema clasificatorio y otras funciones de los numerales; segundo que la función de los plurales se encuentra planteada en este artículo en una forma simple, aunque no expositiva, sino funcional.

La escritura que aparece en este artículo, aplicada al quiché, es la que se emplea en Guatemala en las instituciones oficiales, y es empleada también por el Instituto Lingüístico de Verano; considero más adecuada esta grafía que la propuesta por los lingüistas norteamericanos del Proyecto Marroquín; las aclaraciones con respecto a la escritura son las siguientes:

$\mathrm{v}$, vocal con duración normal, aparecen: a, e, i, o, u.

$\ddot{v}$, vocal corta (para algunos hablantes llegan a ser cortas y abiertas), aparecen: ä, ë, $\ddot{i}$, ö, ü.

$\mathrm{p}$, representa $/ \mathrm{p} /$

b , representa $/ \mathrm{b} /$

$\mathrm{t}$, representa / $\mathrm{t} /$ 


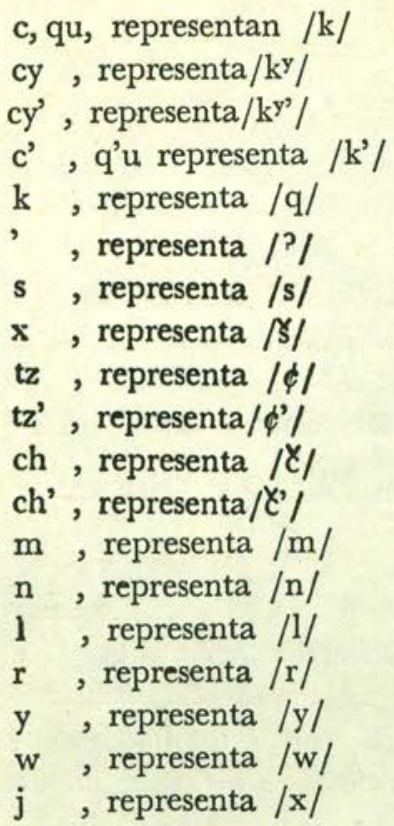

En relación a $/ x /$, o sea " $\mathrm{j}$ ", se presenta el siguiente problema; hay diferencia de pronunciación entre jóvenes y adultos en relación a este fonema; entre los jóvenes la tendencia es a sustituir / $\mathrm{x} /$ por $/ \mathrm{h} /$, y entre los adultos se encuentran estos sonidos pero como alófonos de un mismo fonema; en ambos casos no llega a contrastar.

El fonema $/ r /$, generalmente aparece como una vibrante múltiple sorda, que tiene un alófono vibrante simple sonoro que aparece en posición inicial.

En el quiché de este municipio se encuentran dos tipos de palabras que se inician con cierre glotal: A) las que reciben prefijos o clíticos que hacen que el cierre glotal inicial desaparezca y B) las que mantienen el cierre glotal al recibir afijos pronominales; la categoría A) es común a todas las lenguas mayas; la categoría B) aparece en el quiché de Sajcabajá, como consecuencia de la sustitución que hace este dialecto de la oclusiva posvelar sorda glotalizada /q'/ por cierre glotal; en este caso se conserva el cierre inicial, pero existen en este dialecto, muchos términos o palabras que han perdido en posición inicial, no sólo consonantes sino sílabas completas. Ej:

'akan pierna (en otros dialectos del quiché), en Sajabajá aparece como kan pierna. 


\section{Sistema Numeral}

El sistema numeral, tal como se usa en la actualidad en ese pueblo, es vigesimal, al igual que en el resto de las lenguas mayas, presentando divisiones de grupos numéricos de 1 a 5 , de 6 a 10 , de 11 a 15 y de 16 a 20 . El primer grupo de números (del 1 al 5), se caracteriza por principiar todos ellos con velares y glotales, además de carecer de consonantes posvelares. El grupo de números del 6 al 10 tienen posvelares en tres de ellos, y todos (menos el número 10), se inician con bilabial. En el grupo de 11 a 15, todos llevan el sufijo -lajuj para marcar diez. Del 11 al 20, nuevamente se inician con bilabial, por ser las mismas raíces que marcan los números del 6 al 10 y; para marcar diez, llevan el sufijo -ljuj. Esto no funciona para el veinte. Los cardinales (para cosas en general), sólo pueden llevar como clasificador -b, -ib, -ub, -eb, en los números del 2 al 9, estos clasificadores se usan para marcar cosas en general. Los números cardinales son:

$\begin{array}{rlrl}1 & \text { jun } & 16 & \text { wakljuj } \\ 2 & \text { cyeb } & 17 & \text { wukljuj } \\ 3 & \text { oxib } \sim \text { uxib } & 18 \text { waxakljuj } \\ 4 & \text { cyjeb } \sim \text { cjeb } & 19 & \text { belejljuj } \\ 5 & \text { j'ob } & 20 & \text { juwinak } \\ 6 & \text { wakib } & 21 & \text { juwinak jun } \\ 7 & \text { wukub } & 22 & \text { juwinak cyeb } \\ 8 & \text { waxkib } & 23 & \text { juwinak oxib } \\ 9 & \text { beljeb } & 24 & \text { juwinak cyjeb } \\ 10 & \text { ljuj } & 40 \text { cawinak } \\ 11 & \text { julajuj } & 50 \text { cawinak lajuj } \\ 12 & \text { cablajuj } & 60 \text { oxc'al } \\ 13 \text { oxlajuj } & 70 \text { oxc'al lajuj } \\ 14 & \text { cajlajuj } & 80 & \text { jumuch' } \\ 15 & \text { oljuj } & 100 \text { jun siento }\end{array}$

La formación de números que no son múltiplos de veinte, pero que lo son de diez, se hace sumando a la veintena correspondiente el diez.

Las raíces de los números cardinales, cuando van acompañadas de clasificadores concretos '(no de elementos en general), son las siguientes:

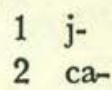


3 ox-

4 caj-

5 j'o-

Estos números no pueden aparecer sin un clasificador específico, y los clasificadores que los posceden son los siguientes.

\section{Clasificadores}

Funcionan como clasificadores algunos nombres, verbos y elementos que marcan tiempo. Aunque trataré después las raíces verbales que funcionan como tales, hay algunos clasificadores propiamente dichos, pero sólo en el aspecto de medidas. Los elementos que encontré que funcionan como clasificadores son los siguientes:

-mö’ para contar puñados

-rok, para pedazos largos y partes de un manojo

-bor, para contar manojos de plantas que han de ser transplantadas, manojos de zacate, ramos de flores y rollos de petates.

-kux, mordidas

-tz'up, chupadas o lamidas

_tuz $\backsim$-tob, chupadas que se le dan a un cigarro

-tun, lienzos de ropa

-kum, tragos

-sa', hileras de cosas tendidas

-'och, vueltas que tienen un rollo

-tzac, para contar cosas cocidas o cocinadas

-pir $\sim$-per, para rebanadas o pedazos

-much', grupos de ochenta unidades, o bien lo que agarran las puntas de los dedos (en cosas diminutas).

-mul, veces

-sut, vueltas

-mlaj, montones grandes

-puk, parvadas o manadas, o grupos de gente

-sol, capas

-'chom, rollos

-tik, pisos

-mutz, puñados

-mutz', cardumen, grupos o puñados de pescados

-xa', pasos

-lajuj, para números del 11 al 14

-ljuj, para números del 15 al 19 
-winak, para contar veintenas

_c'al, para veintenas

$-\mathrm{ub},-\mathrm{ob},-\mathrm{ib},-\mathrm{b}$, para contar objetos en general

La fórmula para el número completo, entre los que usan clasificadores (sólo para los del 1 al 5), sería:

Número $=$ +Núcleo : raíz numeral +Clasificador : clasificador $/$ nombre/raíz verbal.

Ejemplos:

mu'y, juilines o bagres de agua dulce jmutz', un cardumen jmutz' mu'y, un cardumen de juilines camutz' mu'y, dos cardúmenes de juilines oxmutz' mu'y, tres cardúmenes de juilines cajmutz' mu'y, cuatro cardúmenes de juilines j'obmutz' mu'y, cinco cardúmenes de juilines u xa' mnak, un paso de gente (o persona) nmak caxa', dos pasos grandes

\section{Los Ordinales}

1o. nabe

2o. u cab

3o. u rok

4o. u caj

5o. u ro' 6o. u wak

7o. u wuk

8o. u wajxak

9o. u bljej $\sim$ u biljej

último, q'uisbal re

Nótese que, además de ir poseídos por la tercera persona del singular, carecen de clasificadores, al igual que en las otras lenguas mayas.

Los ancianos y las mujeres usan normalmente los ordinales para contar elementos de tiempo (como años, meses, semanas y días). Esto no sucede de una forma mecánica entre los jóvenes varones, que usan indistintamente el sistema de ordinales o el de cardinales para contar los mismos elementos.

\section{Los Clasificadores de días}

Aunque los clasificadores de días se encuentran ligados a tiempo 
pasado y futuro, éstos se comportan de otra forma, ya que nunca aparecen con la raíz numeral de uno, sino que principian con el dos, habiendo nombres que determinan un día en pasado $\mathrm{y}$, otro, para un día en presente o en futuro.

-ij, para contar días en futuro.

-jir, para contar días en pasado.

Ejemplos:

iwir, ayer

cabjir, antier

oxjir, hace tres días

chuek, mañana

c'bij, pasado mañana

oxij, dentro de tres días

\section{Los Numerales y Los Verbos}

Algunas raíces verbales pueden funcionar como clasificadores y en algunos casos un nombre funciona como clasificador de una acción ligada a un verbo; concepción que se hace de acuerdo con su significado, y no de acuerdo con su estructura. La mayoría de los verbos, sin embargo, lleva un número completo ante ellos cuando es necesario contar acciones.

Las raíces verbales que pueden funcionar como clasificadores son las que corresponden a acciones que pueden repetirse de acuerdo con una meta a seguir. Ejemplos:

tic, raíz de sembrar y domesticar

ch'aj, raíz de lavar

jtic, primera siembra

catic, segunda siembra

oxtic, tercera siembra

jch'aj, primera lavada

cach'aj segunda lavada

oxch'aj, tercera lavada

En el caso de tic, la meta sería mantener una buena siembra o lograr una buena cosecha; en el caso de ch'aj, sería mantener un objeto limpio o determinar su antigüedad.

Cuando se trata de acciones que al realizarse constituyen una meta 
por sí misma, se usan los ordinales para contar dichas acciones. Ejemplos:

chnaj, calear o lechar una casa

nabe chnaj, calear o lechar por primera vez

u cab chnaj, calear o lechar por segunda vez

wuch, significa fruta, cara o superficie; se usa para contar cosechas de plantas que pueden dar frutos por un tiempo largo, así como mangos, tomates... etc.

nabe wuch, primera cosecha

u cab wuch, segunda cosecha

$\mathrm{u}$ rox wuch tercera cosecha

Cuando se cuentan acciones que ya se han llevado a cabo de una manera obligatoria, se usan numerales completos (raíz numeral + clasificador), Ejemplo:

'ch'kow, hervir

u camul 'uch'kow, segunda hervor o hierve por segunda vez camul, dos veces

\section{Partitivos}

La reduplicación generalmente marca partitivo, pero esto no sucede con el número uno. Para marcar el partitivo con el número uno se suma al número uno (del sistema cardinal para objetos en general) la raíz de uno pero sin agregar clasificador alguno. Ejemplos:

cacab, de 2 en 2 ó a dos cada uno

ox ox, de 3 en 3 ó a tres cada uno

cajcaj, de 4 en 4 ó a cuatro cada uno

junj, de uno en uno ó a uno cada uno

\section{EL PLURAL}

El plural y el oolectivo, en el quiché de Sajcabajá, se marca al nivel de la estructura de frase, como generalidad, aunque hay aún un corto número de nombres que conserva sufijos pluralizadores. En los verbos generalmente se marca el plural de acuerdo con el pronombre que vaya funcionando como sujeto, y se marca en forma de prefijo. Ejemplos: 


\begin{tabular}{|c|c|c|c|}
\hline Nombres & singular & plural & colectivo \\
\hline piedra & abaj & nc'aj abaj & $\begin{array}{l}\text { yojlic abaj } \\
\text { q'uilaj abaj }\end{array}$ \\
\hline árbol & che' & nc'aj che' & $\begin{array}{l}\text { kum che' } \\
\text { q'uilaj che' }\end{array}$ \\
\hline arena & senyab & nc'aj senyab & q'ui senyab \\
\hline casa & & nc'aj ja & q'uilaj ja \\
\hline flor & cotz'ij & nc'aj cotz'ij & q'uilaj cotz'ij \\
\hline perro & tz'i' & nc'aj tz'i' & q'uilaj tz'i' \\
\hline
\end{tabular}

En construcciones de frase, el plural constituye un elemento por sí solo en la construcción. Ejemplos:

wkan, mi pie nc'aj wkan, mis pies

juybal aw, puerco salvaje nc'aj juybal ak, puercos de monte o salvajes

ati' äc', gallina nc'aj ati' äc', gallinas

El plural generalmente se marca con el adverbio de cantidad nc'aj, o bien q'ui, que sinifica muchos; cuando un nombre va después de un número, que sea mayor a la unidad, el nombre adquiere el carácter de plural; los colectivos se marcan de la misma forma que los plurales, variando solamente el adverbio que los marca.

Existe un prefijo pluralizador $\mathrm{y}_{\rightarrow}$, que funciona con gentilicios o con elementos que vayan precedidos del prefijo aj-, que marca actor u origen. Ejemplos:

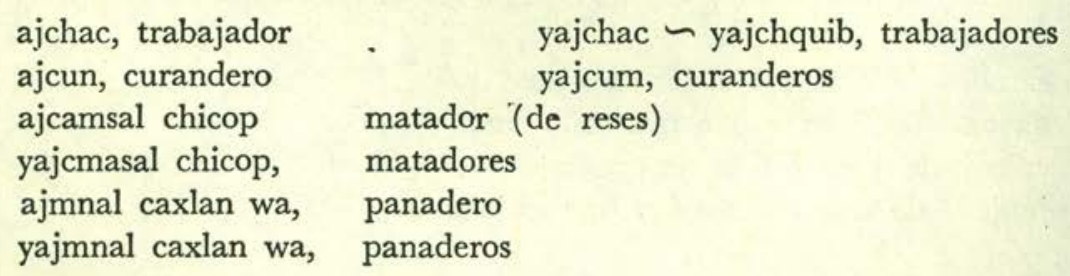

El elemento tak aparece marcando plural, con adjetivos y con nombres; aunque, cuando precede a un nombre, generalmente lleva sentido no sólo de plural, sino también de diminutivo. Ejemplos:

abaj, piedra

ch'in, pequeño

nmak tak winak, personas grandes tak abaj, piedrecitas tak ch'in, pequeños winak, persona 
co'coj tak awaj, animales chiquitos

ch'in tak ja, casitas pequeñas

utz tak ja, casitas bonitas

$\mathrm{u}$ chcäp tak aspeja, pedacitos de vidrio

tak ja, casas o casitas awaj, animal

ja, casa

utz, bueno

aspeja, vidrio

Nótese que el sentido de diminutivo se pierde en este pluralizador, cuando va precedido de un modificador que lleve el sentido contrario, como en el caso de personas grandes. Cuando va precedido de un locativo, también pierde su sentido diminutivo, conservando sólo el de plural. Ejemplos:

txol tak ja, entre las casas

xol tak che', entre los árboles

txol o xol, entre

Cuando el elemento tak precede a una raíz verbo-nominal que se encuentre funcionando como nombre, también lleva el carácter de plural diminutivo. Ejemplos:

cwaj tak chac, quiero trabajitos

cwaj chac, quiero trabajo o quiero trabajar

chac, trabajo o trabajar

Este elemento tak es átono, y podría considerarse como un clítico; aunque tradicionalmente se ha considerado como independiente o preposición.

En los ejemplos antes expuestos podemos ver cómo, al nivel de frase, aparecen en los nombres formas que se pierden cuando van libres de contexto. Así, en el ejemplo "personas grandes" (nmak tak winak), aparece la forma winak para persona, que, en forma independiente, generalmente es mnak. Esto se debe a la tendencia a evitar que en una misma frase aparezcan dos elementos que tengan pérdida de vocal, como lo es mnak, que alterna con winak, persona; nmak que alterna con nimak, grandes. Esta tendencia, por lo tanto, puede ser sólo un elemento de ritmo; pero de ninguna manera constituye una regla.

El plural en los adjetivos puede hacerse de varias formas. Una, marcándolo con un sufijo pluralizador distinto al de los nombres, o bien, con reduplicación parcial o con el elemento tak, que en este caso generalmente va después del adjetivo. Ejemplos: 
nim, grande

co'lic, pequeño

pim, grueso nmak, grandes

co'coj, pequeños

pimtak, gruesos

El sufijo -oj, que aparece en pequeños, es un elemento formativo.

Registré un número regular de nombres que reciben sufijo para marcar el plural. Los nombres que reciben este tipo de afijos pierden elementos. En un caso, pude registrar metátesis con pérdida. Ejemplos:

ala, joven varón

ali, señorita

achi, hombre

el'om, ladrón

ixok, mujer abom, jóvenes varones

iltom, señoritas

achjab, hombres

el'mab, ladrones

ixkib, mujeres

Los sufijos que marcan plural en los nombres, generalmente, son: -eb, -ab, -ib.

\section{Aumentativo}

El elemento que marca el aumentativo es un sufijo átono que va después de un adjetivo. Este mismo elemento, al ir como prefijo de un sustantivo, marca diminutivo. Ejemplos:

utz, bueno

nim, grande

q'ui, mucho

cyeb, caballo

tz'i' perro

cuc, ardilla utzlaj, muy bueno o buenísimo

nimlaj, grandísimo

q'uilaj, muchísimo

lajcyej, caballito

lajtz'i', perrito

lajcuc, ardillita

Como puede verse en el material presentado, el uso de clasificadores en el quiché moderno no se ha perdido, como han planteado algunos investigadores; además, es importante hacer notar que las vocales cortas, que en otros dialectos de esta lengua son frecuentes, en el quiché de Sajcabajá tienden a desaparecer sin dejar rastro. 